\title{
Ant Colony Algorithm for Just-in-Time Job Shop Scheduling with Transportation Times and Multirobots
}

\author{
Fatima El Khoukhi, ${ }^{1,2}$ Tarik Lamoudan, 1,2 \\ Jaouad Boukachour, ${ }^{1}$ and Ahmed El Hilali Alaoui ${ }^{2}$ \\ ${ }^{1}$ CERENE, ISEL, Quai Frissard, BP 1137, 76063 Le Havre Cedex, France \\ ${ }^{2}$ Modelling Laboratory and Scientific Computing, Faculty of Science and Technology, BP 2202, \\ Route D'Imouzzer Fez, Morocco \\ Correspondence should be addressed to Ahmed El Hilali Alaoui, elhilali_fstf2002@yahoo.fr
}

Received 27 March 2011; Accepted 27 April 2011

Academic Editor: S. G. Garcia

Copyright (c) 2011 Fatima El Khoukhi et al. This is an open access article distributed under the Creative Commons Attribution License, which permits unrestricted use, distribution, and reproduction in any medium, provided the original work is properly cited.

\begin{abstract}
Handling rapidly evolving technology and almost daily changes in demand and customer satisfaction, while maintaining competitiveness in a highly competitive environment, requires good coordination and planning of both production and logistics activities on the shop floor, namely: machines and tools. The goal is to optimize costs and reduce delivery lead times in order to provide the customer just in time; we focus on the job shop scheduling problem (JSSP), which is one of the most complex problems encountered in real shop floor. In this paper, we study a generalized (JSSP) including transportation times and a set of additional constraints on the number of transporter vehicles and their multiple transfer capabilities and also on the limited capacity of input/output of machines. The objective is to minimize in one hand tardiness and earliness penalties on delays and advances compared to the lead-time delivery of finished jobs and on the other hand the number of empty moves of transporter vehicles.
\end{abstract}

\section{Introduction}

Scheduling may be defined as the allocation of resources to tasks overtime to optimize a criteria. From the view point of production scheduling, the resources and tasks are commonly referred to a machines and jobs and the commonly used criteria is the completion times of jobs (makespan). In this paper, we study a variant of JSSP where the jobs have to be transported between the machines by one or several transporter vehicles. At JSSP, each job has its own processing order through the machines (a sequence of operations). Each operation must be processed on a fixed machine on which it has to be processed without preemption for a given 
duration. Each machine can process just one operation at each time and each job can be performed on one machine at each time. Additionally, transportation times are considered.

The JSSP is known to be strongly NP-hard and exists in several variants according to the additional constraints considered such as, the classical problem [1], the flexible one [2], the cyclic problem [3], the dynamic job shop scheduling [4], the stochastic case [5], the reentrant problem [6], the case with separable setup time [7], the blocking job shop [8].

The JSSP, classified NP-complete, has attracted many researchers, $[1,7,9,10]$. Including the transportation times between different machines, gives rise to a number of variants of the classical JSSP, particularly those concerned with job shops and transportation times. According to [11], there are two kinds of problems (1) the first one occurs in production manufacturing, when we need to transport jobs between machines and (2) the second one generally occurs at the level of delivery of products to customers. In many industries, production and delivery systems are integrated, with finished products being transferred from a manufacturing execution service to a customer delivery service. In this case, the most common form of delivery to the customer is by vehicle, with the makespan being given by the last delivery date.

In both types, the commonly used objective is to find a scheduling solution which minimizes the makespan. Related works that examine the JSSP with the transportation times (either type 1 or 2), include the works of [12-17]. Additionally, Yuan et al. [18] studied the complexity of flow shop problem with transportation times, in order to minimize the makespan. Brucker et al. [19] considered the job shop scheduling with limited capacity buffers. Finally, Caumond et al. [20] give a linear formulation taking into account the maximum number of jobs allowed in the system, limited input/output buffer capacities, empty trips and no-move-ahead trips simultaneously.

In this paper, our contribution presents one generalization version of the JSSP with transportation times, integrating the different additional constraints mentioned previously, including the existence of several transporter vehicles with multiple transfer capacity in the shop. Moreover, our model incorporates other constraints related to storage buffers associated with each machine taking into account the case of limited buffer spaces. In our case, we study specially the transportation activities, the empty or not. To achieve a just in time (JIT) production [21], the goal is to minimize the earliness and tardiness penalties with regard to the delivery deadline of finished products, as well as penalties on empty activities.

The remainder of the paper is organized as follows. In Section 2, we give a formal definition of the considered scheduling problem and state some additional assumptions. Section 3 deals with the mathematical modeling of the problem and Section 4 presents our method of resolution. The computational results can be found in Section 5. Finally, a conclusion is given in Section 6.

\section{Problem Context}

In this work, we study the JSSP with transportation times taking into account the following constraints:

(i) JIT scheduling in order to respect the delivery times (latest completion time) imposed by customers,

(ii) a set of homogenous transporter vehicles, 
(iii) a transporter vehicle has a finite capacity of transfer more than one (in terms of a number of tasks they can carry),

(iv) a station $=\{$ input + machine + output $\}$ (see Figure 1),

(v) a station input/output buffers has a limited capacity, not necessary the same for both storage spaces,

(vi) two deposits, the initial one for arrival jobs and the final one for finished jobs.

We show below a mathematical formulation of the problem, with all basics constraints of the classical JSSP, and additional transport constraints concerning the transporter vehicles, their transfer capacity and the limited capacity of storage buffers associated with each machine.

\section{Mathematical Formulation}

\subsection{Classical Data}

(i) $m$ : number of machines,

(ii) $M=\left\{M_{1}, M_{2}, \ldots, M_{m}\right\}$ : set of machines,

(iii) $n$ : number of jobs,

(iv) $J=\left\{J_{1}, J_{2}, \ldots, J_{n}\right\}$ : set of jobs,

(v) $O_{i \sigma_{i k}}$ : the $k$ th task of the job $i$,

(vi) $\sigma_{i k}$ : the machine required by the task $O_{i \sigma_{i k}}$,

(vii) $J_{i}=\left\{O_{i \sigma_{i 1}}, O_{i \sigma_{i 2}}, \ldots, O_{i \sigma_{i i_{i}}}\right\}$ : technological Sequence associated with the job $i$, with $n_{i}$, the number of operations of the job $i$,

(viii) $P_{i}^{\sigma_{i k}}$ : processing time of the operation $O_{i \sigma_{i k}}$,

(ix) $r_{i}^{\sigma_{i k}}$ : earliest starting time of the operation $O_{i \sigma_{i k}}$,

(x) $d_{i}^{\sigma_{i k}}$ : due date of the operation $O_{i \sigma_{i k}}$,

(xi) $\alpha_{i}^{k}$ : earliness Penalty of the task with regard to its delivery deadline,

(xii) $\beta_{i}^{k}$ : tardiness Penalty of the task with regard to its delivery deadline.

\subsection{Classical Decision Variables}

(i) $C_{i}^{U}$ : actual finish date of job $i$ on machine $U$, with $C_{i}^{\sigma_{i k}}$ the date of last operation $O_{i \sigma_{i k}}$ on the required machine $\sigma_{i k}$,

(ii)

$$
\lambda_{i}^{k}= \begin{cases}1, & \text { if } C_{i}^{\sigma_{i k}} \leq d_{i}^{\sigma_{i k}}, \\ 0, & \text { otherwise }\end{cases}
$$

The previous equation is the binary variable to record the earliness or lateness associated with delivery delay of operation $O_{i \sigma_{i k}}$. 


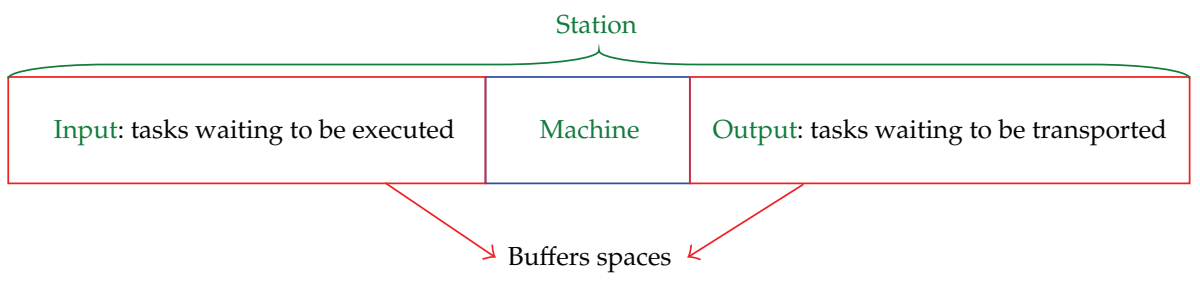

Figure 1: Station model used in this paper.

\subsection{Classical Constraints}

The first constraint ensures that the first operations of the jobs must be processed respecting their corresponding earliest starting times

$$
C_{i}^{\sigma_{i 1}}-P_{i}^{\sigma_{i 1}} \geq r_{i}^{\sigma_{i 1}}, \quad \forall i=1, \ldots, n
$$

The second constraint guarantees the respects of the precedence sequences constraints predefined between the tasks of the same job

$$
C_{i}^{\sigma_{i k}} \leq C_{i}^{\sigma_{i k+1}}-P_{i}^{\sigma_{i k+1}}, \quad \forall i=1, \ldots, n, \forall k=1, \ldots,\left(n_{i}-1\right)
$$

The disjunction at the level of machines is specified in the third constraint

$$
C_{E_{i j}}^{U}-P_{E_{i j}}^{U} \geq C_{i+j-E_{i j^{\prime}}}^{U}, \forall i<j \in O_{U}, \forall U \in M
$$

with

$$
E_{i j}= \begin{cases}j, & \text { if job } i \text { precedes } j, \\ i, & \text { otherwise, }\end{cases}
$$

$$
O_{u}=\{\text { set of jobs requiring the machine } U\} .
$$

Remark 3.1. Our constraint is an improvement of the constraint of disjunction appearing in the literature (see [22]) taking the following general form:

$$
C_{j}^{U}-C_{i}^{U}+H\left(1-a_{i j u}\right) \geq P_{j}^{U}, \quad \forall i, j=1, \ldots, n, \forall U \in M,
$$

with

$$
a_{i j U}= \begin{cases}1, & \text { if job } i \text { precedes } j \text { on the machine } U, \\ 0, & \text { otherwise, }\end{cases}
$$

$$
H \text { : a big value. }
$$




\subsection{Additional Data, Variables, and Constraints}

In manufacturing production, a transporter vehicle undertaken to perform any or all of the following activities: loading the input of a station, unloading the output of a station and/or removing a task from one a station to another.

In general, where the transporter vehicle can carry only one task at a time, two cases are presented:

(i) if the transporter vehicle moves from a source machine to a destination one in order to load it, then a transportation time is considered, it is a transport activity,

(ii) if the transporter vehicle moves from a source machine to a destination one without loading it, an empty travel time is considered; it is an empty movement activity.

Here, we consider the problems that the transporter vehicles can carry more than one task at a time and for each machine there are two associated storage buffers input/output, where tasks can wait for their execution or transportation. In the remainder of this paper, we introduce the following definitions:

(1) a transporter vehicle performs a "transport activity" if it moves in a direct way from a station to another one to load it,

(2) a transporter vehicle performs an "empty movement activity" (even it is charged), if it moves in a direct way from a source station to a destination one without loading it.

\subsubsection{Additional Data}

(1) R: number of transporter vehicles in the workshop (assuming more than one),

(2) $S=\left\{S_{1}, S_{2}, \ldots, S_{m}\right\}:$ set of stations,

(3) $C_{r}$ : capacity of the transporter vehicle $r$ (in terms of numbers of tasks to carry),

(4) $\varepsilon_{c}$ : loading time of one or several tasks on the input of a station,

(5) $\varepsilon_{d}$ : unloading time of one or several tasks from the output of a station,

(6) Cout $(U)$ : output Capacity of the machine $U$,

(7) Cin $(U)$ : input Capacity of the machine $U$,

(8) T: big value representing an upper bound for the production horizon,

(9) $\delta_{s d}$ : transportation time from a station $s$ to a station $d$,

(10) $\delta_{s d}^{\prime}$ : empty movement activity from a station $s$ to a station $d$. 


\subsubsection{Additional Decision Variables}

We use additional decision variables to express the objective function and additional constraints on the transport of tasks between machines, and the activities of transporter vehicles

$$
\begin{gathered}
t_{i \sigma_{i k}}: \text { starting transportation time of task } O_{i \sigma_{i k}}, \\
x_{i \sigma_{i k}}^{r}= \begin{cases}1, & \text { if the task } O_{i \sigma_{i k}} \text { is transported by the transporter vehicle } r, \\
0, & \text { otherwise, }\end{cases} \\
S_{i \sigma_{i k}}^{t}= \begin{cases}1, & \text { if the task } O_{i \sigma_{i k}} \text { is transported in time } t, \\
0, & \text { otherwise, }\end{cases} \\
V_{r}^{a}= \begin{cases}1, & \text { if the transporter vehicle is in "empty movement activity, } \\
0, & \text { if the transporter vehicle is in "transport activity." }\end{cases}
\end{gathered}
$$

\subsubsection{Additional Constraints}

A task can be handled by only one transporter vehicle at a time

$$
\sum_{r=1}^{R} x_{i \sigma_{i k}}^{r}=1, \quad \forall i=1, \ldots, n, \forall k=1, \ldots, n_{i}
$$

Each job requires $n_{i}+1$ tasks of transport

$$
\sum_{r=1}^{R} \sum_{k=0}^{n_{i}+1} x_{i \sigma_{i k}}^{r}=n_{i}+1, \quad \forall i=1, \ldots, n
$$

$\sigma_{i 0}$ and $\sigma_{i\left(n_{i}+1\right)}$ are two fictitious machines representing the initial and the final deposit of the jobs in the workshop.

A task can be performed only after its transport

$$
C_{i}^{\sigma_{i k+1}}-P_{i}^{\sigma_{i k+1}} \geq t_{i \sigma_{i k}}+\delta_{S\left(\sigma_{i k}\right)} S\left(\sigma_{i k+1}\right)+\varepsilon_{d}, \quad \forall i=1, \ldots, n, \forall k=1, \ldots, n_{i}
$$

where $S\left(\sigma_{i k}\right)$ is the station associated with the machine $\sigma_{i k}$

Each task can be transported only after its completion

$$
t_{i \sigma_{i k}} \geq C_{i}^{\sigma_{i k}}+\varepsilon_{c}, \quad \forall i=1, \ldots, n, \forall k=1, \ldots, n_{i} .
$$


The current capacity of the transporter vehicle $r$ at time $t$ (noted $C_{r}^{t}$ ) must not exceed its capacity

$$
C_{r}^{t}=\sum_{i=1}^{n} \sum_{k=1}^{n_{i}} S_{\sigma_{i k}}^{t} x_{i \sigma_{i k}}^{r} \leq C_{r}, \forall r=1, \ldots, R, \forall t=0, \ldots, T
$$

The load of a transporter vehicle at each time $t$ must not exceed its current available capacity $\left(C_{r}-C_{r}^{t}\right)$,

$$
\sum_{i=1}^{n} \sum_{k=1}^{n_{i}} \mathrm{out}_{\sigma_{i k}}^{t} x_{i i_{i k}}^{r} \leq C_{r}-C_{r}^{t}, \quad \forall r=1, . ., R, \forall t=0, \ldots, T
$$

such as

$$
\text { out }_{\sigma_{i k}}^{t}= \begin{cases}1, & \text { if } C_{i}^{\sigma_{i k}} \leq t<t_{i \sigma_{i k+1}} \\ 0, & \text { otherwise. }\end{cases}
$$

The limited capacity of the outputs of machines is respected at every time $t$

$$
\sum_{i=1}^{n} \sum_{k=1 / \sigma_{i k}=u}^{n_{i}} \text { out }_{\sigma_{i k}}^{t} \leq C_{\text {out }}(u), \quad \forall u \in M, \forall t=0, \ldots, T .
$$

The limited capacity of the inputs of machines is respected at every time $t$

$$
C_{i n}^{t}(u)=\sum_{i=1}^{n} \sum_{k=\frac{1}{\sigma_{i k}}=u}^{n_{i}} \operatorname{int}_{\sigma_{i k}}^{t} \leq C_{i n}(u) \quad \forall u \in M \forall t=0, \ldots, T,
$$

with

$$
\operatorname{int}_{\sigma_{i k}}^{t}= \begin{cases}1, & \text { if } t_{i \sigma_{i k}}+\delta_{S\left(\sigma_{i(k-1)}\right) S\left(\sigma_{i k}\right)}+\varepsilon_{d} \leq t<C_{i}^{\sigma_{i k}}-P_{i}^{\sigma i k} \\ 0, & \text { otherwise }\end{cases}
$$

where $C_{i n}^{t}(u)$ is the current capacity of the input associated with the machine $u$.

At every moment, the transporter vehicles should serve only the available inputs of the machines

$$
\sum_{r=1}^{R} \sum_{i=1}^{n} \sum_{k=1 / \sigma_{i k}=u}^{n_{i}} S_{i \sigma_{i k}}^{t} x_{i \sigma_{i k}}^{r} \leq C_{i n}(u)-C_{i n}^{t}(u), \quad \forall u \in M, \forall t=0, \ldots, T .
$$


The following recurrent formula computes the completion time of service of transporter vehicles on every machine which they serve, noted by $F_{r}^{s}$,

$$
F_{r}^{s}=F_{r}^{s-1}+v_{a}^{r}\left(\delta_{(s-1) s}^{\prime}+\varepsilon_{c}\right)+\left(1-v_{a}^{r}\right)\left(\delta_{(s-1) s}+\varepsilon_{d}+f_{r}^{s} \varepsilon_{c}\right), \quad \forall r=1, \ldots, R, \forall s \in S,
$$

with

$$
f_{r}^{s}= \begin{cases}1, & \text { if the transporter vehicle unloads the station } s \\ 0, & \text { otherwise }\end{cases}
$$

Finally, we express the separation time restrictions due to the type of transporter vehicle activities, "transport activity" (transportation time $\delta_{s d}$ ) or "empty movement activity" (time of empty travel time $\delta_{s d}^{\prime}$ )

$$
\begin{array}{r}
v_{a}^{r}\left(F_{r}^{s}+\delta_{s d}^{\prime}-F_{r}^{d}\right)+\left(1-v_{a}^{r}\right)\left(F_{r}^{s}+\delta_{s d}+\varepsilon_{d}-F_{r}^{d}\right) \leq 0 \\
\forall r=1, \ldots, R, \forall s, d \in S, \forall a \in \text { Activities. }
\end{array}
$$

\subsection{Objective Function}

The objective function seeks to minimize the sum of earliness and tardiness penalties with regard to the delivery deadlines of the finished jobs, as well as the sum of the penalties on the empty movement activities of the transporter vehicles

$$
\operatorname{Min} \sum_{i=1}^{n} \sum_{k=1}^{n_{i}}\left(-\alpha_{i}^{k}\right)^{\lambda_{i}^{k}}\left(\beta_{i}^{k}\right)^{\left(1-\lambda_{i}^{k}\right)}\left(c_{i}^{\sigma_{i k}}-d_{i}^{\sigma_{i k}}\right)+\sum_{r=1}^{R} \sum_{a \in \text { Activities }} m_{a}^{r} v_{a}^{r}
$$

such as

$\alpha_{i}^{k}$ : earliness penalty on the task $O_{i \sigma_{i k}}$,

$\beta_{i}^{k}$ : tardiness penalty on the task $O_{i \sigma_{i k}}$,

$m_{a}^{r}$ : penalty on an "empty movement activity".

\section{Ant Colony Algorithm}

According to our previous work [23-25], we found that ant colony algorithm is better than genetic algorithm in terms of solutions even sometimes with more execution time.

For the resolution of the generalized version of the job shop with transportation times studied in this paper, we propose a resolution algorithm based on ant colony optimization that we denote by "ACOJST" (ant colony optimization for job shop with transportation times), in order to generate the starting times of operations, as well as their starting transportation times.

Ants move on the problem graph (see Figure 2). Each node is associated with a station $\mathrm{S}_{k}$ which is composed of the machine $\mathrm{M}_{k}$ and its input $\mathrm{I}_{k}$ and output $\mathrm{O}_{k}$. A dotted arc 
represents a connection between the deposits, respectively, initial and final towards the various stations. A continuous arc connecting two stations represents the transfer path for the transporter vehicles. Finally, a quantity of pheromone $\tau_{S_{k} S_{h}}$ is associated with each arc of the proposed graph; this quantity simulates the density of transfer between the stations.

Our algorithm contains two main stages, the initial deposit stage and the production one. The first stage initializes transporter vehicles by the first operations of jobs according to a heuristic that we developed. The heuristic takes into account the number of transporter vehicles, their capacity and the total capacity of the initial deposit (in terms of the number of operations to be transported). Then, the loaded transporter vehicles are directed to stations, according to a priority rule, priority is giving to the destination station for which each transporter vehicle will carry a maximum block of tasks in the minimum time. Moreover, to manage the transporter vehicles movements inside of the shop, we use a heuristic to avoid conflict that may be caused by the arrival of more than one transporter vehicle to the same station at the same time.

During the production stage, we choose a task from the inputs buffer to be executed whenever the station is free. Managing the communication with every transporter vehicle with an input to load or an output to unload, and the need to continually choose the next station to be served are all the decisions that we take by appropriate rules. During the cycle of production, there are two steps.

(1) Associate with the input of every station, a fictitious immovable ant called "ant machine", is free to choose the task $O_{i \sigma_{i k}}$ to be executed whenever its required machine $\sigma_{i k}$ is available. The choice of this task is based on a priority rule favoring the task with the maximum ratio $\operatorname{rap}_{i k}(t)$ in $\sigma_{i k}$ :

$$
O_{i \sigma_{i k}}=\underset{\substack{O_{j \sigma_{j h}} \in \operatorname{Input}_{\sigma_{i k}}^{t} \\\left(j=1, \ldots, n, h=1, \ldots, n_{j}\right)}}{\arg \max }\left\{\operatorname{rap}_{j h}(t)\right\},
$$

where

$$
\operatorname{rap}_{i k}(t)=\frac{p_{i}^{\sigma_{i k}}}{\sum_{h=1}^{n_{i}} p_{i}^{\sigma_{i h}}}, \quad \forall O_{i \sigma_{i k}} \in \operatorname{Input}_{r}^{t}
$$

Input $_{\sigma_{i k}}^{t}=\left\{\right.$ tasks belonging in input of machine $\sigma_{i k}$ at the moment $\left.t\right\}$.

(2) In addition, create a family of mobile ants called "ants' robots", which help transporter vehicles to choose the next station to be served by distinguishing two scenarios.

(i) If the transporter vehicle is carrying one or several finished jobs, then facilitating the movement towards the final deposit.

(ii) If it does not carry any finished job then, the choice of the next station is made according to a transition rule making a compromise between the fact of facilitating the least loaded input and the most loaded output (to guarantee that the transporter vehicle serves at most, the chosen station). The rule, that we introduced, distinguishes between two scenarios, the quantity transported by the transporter vehicle overtake or not a threshold.

(a) If the capacity of a transporter vehicle exceeds this threshold, we select stations corresponding to the tasks transported by the chariot of this transporter 


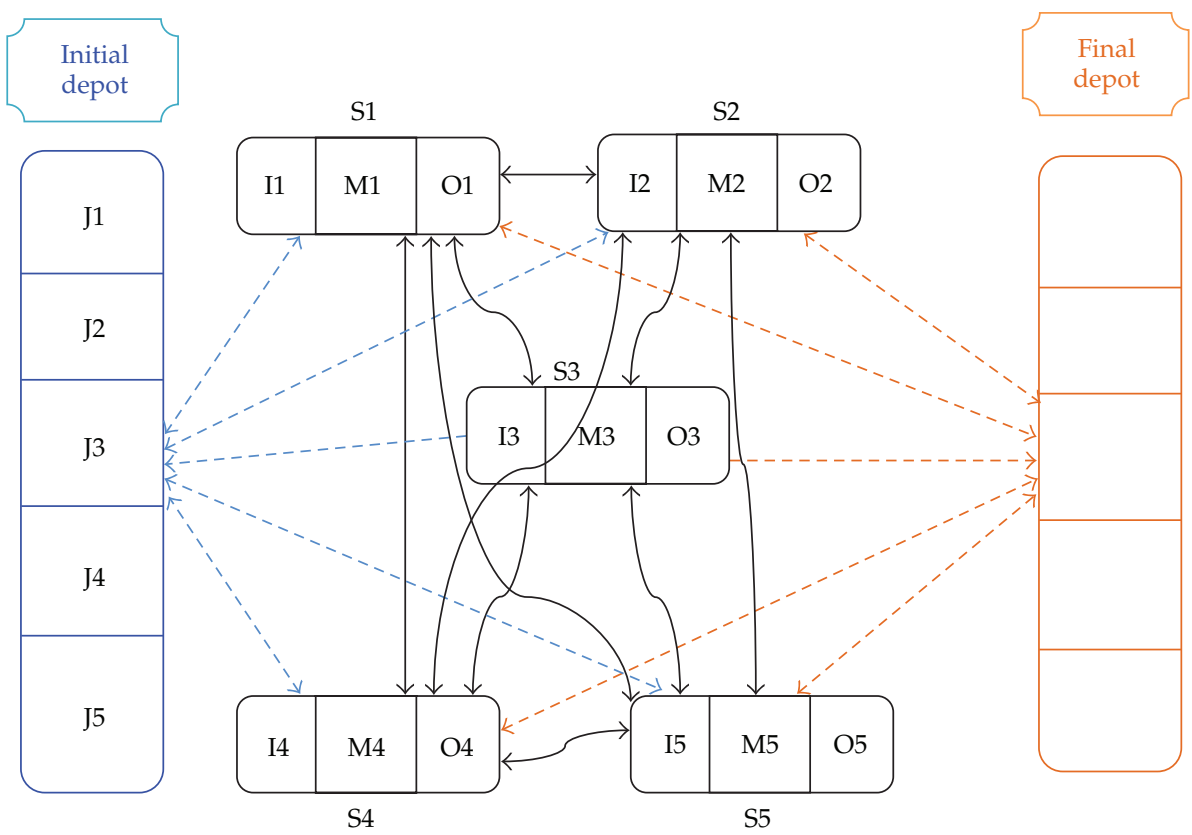

Figure 2: Problem graph.

vehicle, in which case the station to be served is chosen according to the following transition rule:

$$
d(t)=\underset{s \in \text { Chario }(r)}{\arg \max }\left(\tau_{h s}\right)^{\alpha} q_{1}(\beta, \gamma) q_{2}(\theta, \psi) q_{3} .
$$

(b) If the capacity of the studied transporter vehicle does not exceed this threshold, all the stations of the workshop are considered, in this case the station to be served is chosen according to the following transition rules:

$$
d(t)=\underset{s \in S}{\arg \max }\left(\tau_{h s}\right)^{\alpha} q_{1}(\beta, \gamma) q_{2}(\theta, \psi) q_{3},
$$

with:

$$
\begin{gathered}
q_{1}(\beta, \gamma)=\left(1-\frac{1}{\left(\operatorname{Cin}(s)-\operatorname{Cin}^{t}(s)\right)+1}\right)^{\beta}\left(\frac{1}{\left(\operatorname{Cou}(s)-\operatorname{Cou}^{t}(s)\right)+1}\right)^{\gamma} \\
q_{2}(\theta, \psi)=\left(\frac{1}{\delta_{c s}+1}\right)^{\theta}\left(\frac{1}{\delta_{c s}^{\prime}+1}\right)^{\psi} \\
q_{3}=\left(\operatorname{card} \_b l o c(r, s)\right)(\text { tmachine }(s))\left(\frac{1}{F s(s)}\right) .
\end{gathered}
$$


card_bloc $(r, s)$ : number of tasks transported by the transporter vehicle $r$ towards the station $s$ tmachine(s): Total duration of activities of the station $s$ during the horizon of production $\alpha, \beta, \gamma, \theta$, and $\psi$ : These parameters express the compromise between the rate of pheromone, load of the input (less loaded) the load of the output (more loaded, see saturated), the transportation times and the empty movements (minimal).

The goal is to reduce the empty travel and to minimize the transportation times while taking into account the quantity of pheromone, in order to minimize the makespan.

(3) Pheromone can be update locally or globally:

(i) The local update is performed after every transfer of an "ant robot" from a source station $c$ to a destination one $d$, according to the following formula:

$$
\tau_{c d}(k+1)=\tau_{c d}(k)+\tau_{0}\left(1-\rho_{0}\right) .
$$

(ii) The global update is performed at the level of the best solution obtained after each cycle of the algorithm, according to the following formula:

$$
\tau_{m(i) m(i+1)}(k+1)=\tau_{m(i) m(i+1)}(k)+\tau_{0}\left(1-\rho_{0}\right)
$$

(for each pair of successive operation of the best solution),

with:

(a) $m(i)$ : request machine of the task number $i$,

(b) $\tau_{s_{1} s_{2}}(k)$ : quantity of pheromone in the arc connecting the two stations $s 1$ and $s 2$, in the cycle $k$ of the algorithm,

(c) $\tau_{s_{1} s_{2}}(k+1)$ : quantity of pheromone in the arc connecting the two stations $s 1$ and $s 2$, in the current iteration, after update,

(d) $\tau_{0}$ : initial quantity of pheromone,

(e) $\rho_{0}$ : rate of evaporation.

\subsection{Algorithm Description}

In Algorithm 1, we summarize the different steps of our ant colony algorithm with:

(i) $m(i)$ : the request machine of the task $i$,

(ii) initial_deposit: initial deposit of the workshop,

(iii) Chario $(r)$ : set of tasks transported by the transporter vehicle number $r$,

(iv) $c(r, t)$ : current position,

(v) $d(r, t)$ : destination position of the transporter vehicle number $r$ at the moment $t$,

(vi) N1 max and N2 max: stop tests,

(vii) $\tau_{0}$ : initial quantity of pheromone, 


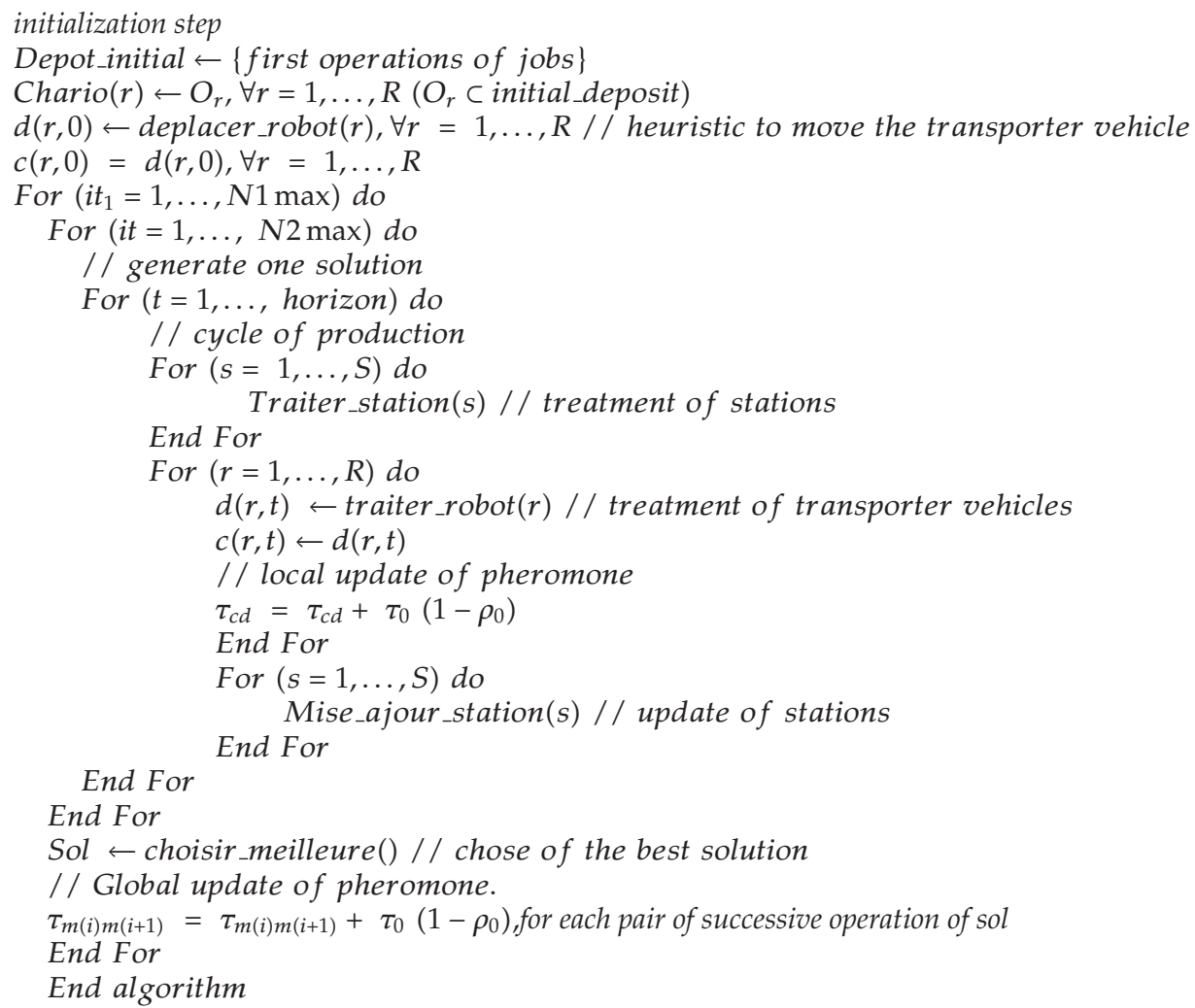

Algorithm 1

(viii) $\rho_{0}$ : rate of evaporation,

(ix) $\tau_{c d}$ : quantity of the pheromone in the arc connecting the two stations $c$ and $d$,

(x) $S$ : number of stations,

(xi) $R$ : number of robots.

\section{Computational Results}

We validate our approach against two types of benchmarks from literature:

(i) 1st type instances: those of Philippe Lacomme, downloadable from the following web page: http://www.isima.fr/ lacomme/Job_Shop_TL.html, for the case of a single transporter vehicle with a capacity of transition equal to 1 ,

(ii) 2nd type instances: we change benchmarks from instances of type 1 to have several transporter vehicles, by changing the number of transporter vehicles, their capacity of transfer and the capacity of the spaces of input/output storages. 
Table 1: Case of a single transporter vehicle.

\begin{tabular}{lllllccc}
\hline Instances & $n$ & $m$ & LB & UB & Lacomme solution & ACOJST & DEV $(\%)$ \\
\hline LT133 & 3 & 5 & 178 & 190 & 178 & 192 & 7.8 \\
LT144 & 4 & 5 & 214 & 224 & 214 & 233 & 8,8 \\
LT155 & 5 & 5 & 248 & 272 & 254 & 271 & 9.2 \\
LT233 & 3 & 5 & 148 & 148 & 148 & 148 & 0 \\
LT244 & 4 & 5 & 160 & 164 & 160 & 167 & 4.3 \\
LT255 & 5 & 5 & 169 & 175 & 169 & 170 & 0.5 \\
LT333 & 3 & 5 & 199 & 211 & 199 & 209 & 5 \\
LT344 & 4 & 5 & 205 & 216 & 205 & 213 & 3.9 \\
LT355 & 5 & 5 & 210 & 227 & 210 & 218 & 3.8 \\
LT433 & 3 & 5 & 271 & 324 & 271 & 350 & 29.1 \\
LT444 & 4 & 5 & 353 & 418 & 353 & 431 & 22 \\
LT455 & 5 & 5 & 426 & 520 & 431 & 501 & 17.6 \\
LT533 & 3 & 5 & 365 & 474 & 365 & 516 & 41.3 \\
LT544 & 4 & 5 & 477 & 612 & 477 & 629 & 31.8 \\
LT555 & 5 & 5 & 585 & 650 & 585 & 731 & 24.9 \\
\hline
\end{tabular}

DEV: deviation of the solution given by ACOJST from LB.

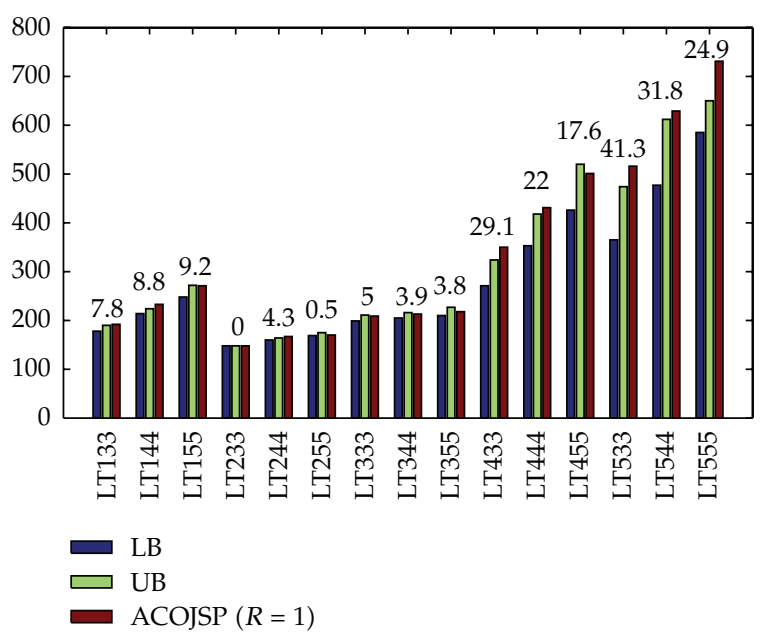

Figure 3: Case of a single transporter vehicle.

Tests were performed on a Pentium (R) Dual-Core CPU E5200 @ 2, 50 GHz, 1 Go RAM. The number of jobs is between three and five and the number of machines was fixed at four, to which we add two fictitious machines represent the initial and final deposits.

The results below are obtained with the capacity of the transporter vehicle between 1 and 2 and the capacity of the input and the output buffer is fixed to 2 . The LB and UB present, respectively, the best known solutions (BKS) obtained by the branch-and-bound procedure coupled with a discrete event simulation model (called B\&B/simulation framework) [15] and by the procedure $B \& B[20]$, that extends the $B \& B /$ simulations. 
Table 2: The results of this comparison.

\begin{tabular}{|c|c|c|c|c|c|c|c|c|}
\hline Instances & LB & UB & $\begin{array}{c}\text { ACOJST } \\
\text { (input management heuristic) }\end{array}$ & FIFO & LIFO & LPT & $\mathrm{SPT}$ & Argmin \\
\hline LT133 & 178 & 190 & 192 & 193 & 192 & 192 & 192 & 193 \\
\hline LT144 & 214 & 224 & 233 & 233 & 233 & 233 & 233 & 233 \\
\hline LT155 & 248 & 272 & 271 & 271 & 271 & 271 & 271 & 271 \\
\hline LT233 & 148 & 148 & 148 & 148 & 148 & 148 & 148 & 152 \\
\hline LT244 & 160 & 164 & 167 & 167 & 164 & 164 & 167 & 164 \\
\hline LT255 & 169 & 175 & 170 & 183 & 182 & 187 & 184 & 183 \\
\hline LT333 & 199 & 211 & 209 & 213 & 211 & 214 & 214 & 212 \\
\hline LT344 & 205 & 216 & 213 & 219 & 219 & 219 & 219 & 219 \\
\hline LT355 & 210 & 227 & 218 & 228 & 228 & 228 & 229 & 228 \\
\hline LT433 & 271 & 324 & 350 & 350 & 350 & 350 & 351 & 350 \\
\hline LT444 & 353 & 418 & 431 & 431 & 431 & 431 & 431 & 431 \\
\hline LT455 & 426 & 520 & 501 & 501 & 501 & 501 & 501 & 501 \\
\hline LT533 & 365 & 474 & 516 & 516 & 516 & 516 & 516 & 516 \\
\hline LT544 & 477 & 612 & 629 & 629 & 629 & 629 & 629 & 629 \\
\hline LT555 & 585 & 650 & 731 & 731 & 731 & 731 & 731 & 731 \\
\hline
\end{tabular}

Table 3: Case of a set of transporter vehicles.

\begin{tabular}{lccccccc}
\hline Instances & $n$ & $m$ & LB & $\begin{array}{c}\text { Lacomme solution } \\
(R=1)\end{array}$ & $\begin{array}{c}\text { ACOJSP } \\
(R=1)\end{array}$ & $\begin{array}{c}\text { ACOJSP } \\
(R=1)\end{array}$ & $\begin{array}{c}\text { ACOJSP } \\
(R=4)\end{array}$ \\
\hline LT133 & 3 & 5 & 178 & 178 & 130 & 117 & 117 \\
LT144 & 4 & 5 & 214 & 214 & 140 & 129 & 132 \\
LT155 & 5 & 5 & 248 & 254 & 147 & 131 & 125 \\
LT233 & 3 & 5 & 148 & 148 & 144 & 145 & 145 \\
LT244 & 4 & 5 & 160 & 160 & 142 & 145 & 145 \\
LT255 & 5 & 5 & 169 & 169 & 149 & 146 & 146 \\
LT333 & 3 & 5 & 199 & 199 & 200 & 200 & 212 \\
LT344 & 4 & 5 & 205 & 205 & 205 & 205 & 205 \\
LT355 & 5 & 5 & 210 & 210 & 204 & 204 & 204 \\
LT433 & 3 & 5 & 271 & 271 & 203 & 158 & 144 \\
LT444 & 4 & 5 & 353 & 353 & 250 & 172 & 159 \\
LT455 & 5 & 5 & 426 & 431 & 264 & 198 & 173 \\
LT533 & 3 & 5 & 365 & 365 & 293 & 230 & 198 \\
LT544 & 4 & 5 & 477 & 477 & 363 & 242 & 231 \\
LT555 & 5 & 5 & 585 & 585 & 374 & 285 & 223 \\
\hline
\end{tabular}

$R$ : the number of transporter vehicles.

\subsection{Transporter Vehicles with a Capacity Equal to 1}

\subsubsection{Use of a Single Transporter Vehicle}

For the case of single transporter vehicle, the results obtained by ACOJST algorithm (see Table 1 and Figure 3 ) show a small deviation $(\leq 10 \%)$ for the first instances (LT133-LT355), for example, for the instance LT233 and LT255, our solutions are identical or nearly identical to the corresponding LB bounds. On the other hand, the deviations increase $(>10 \%)$ for the 
Table 4: (a) Case of 3 transporter vehicles with capacity equal to 2. (b) Case of 3 transporter vehicles with capacity equal to 3 .

(a)

\begin{tabular}{lccccc}
\hline Instances & $n$ & $M$ & $\begin{array}{c}\text { ACOJSP } \\
(R=3, \text { Cap }=1)\end{array}$ & $\begin{array}{c}\text { ACOJSP } \\
(R=3, \text { Cap }=2)\end{array}$ & Gap $(\%)$ \\
\hline LT133 & 3 & 5 & 117 & 126 & 7.7 \\
LT144 & 4 & 5 & 129 & 135 & 4.7 \\
LT155 & 5 & 5 & 131 & 134 & 2.3 \\
LT233 & 3 & 5 & 145 & 151 & 4.1 \\
LT244 & 4 & 5 & 145 & 148 & 2.1 \\
LT255 & 5 & 5 & 146 & 148 & 1.4 \\
LT333 & 3 & 5 & 200 & 203 & 1.5 \\
LT344 & 4 & 5 & 205 & 205 & 0.0 \\
LT355 & 5 & 5 & 204 & 205 & 0.5 \\
LT433 & 3 & 5 & 158 & 185 & 17.1 \\
LT444 & 4 & 5 & 172 & 189 & 9.9 \\
LT455 & 5 & 5 & 198 & 177 & -10.6 \\
LT533 & 3 & 5 & 230 & 263 & 14.3 \\
LT544 & 4 & 5 & 242 & 257 & 6.2 \\
LT555 & 5 & 5 & 285 & 264 & -7.4 \\
\hline
\end{tabular}

$R$ : the number of transporter vehicles.

Cap: the capacity of transfer of a transporter vehicle.

$\mathrm{Gap}=[(B 2-B 1) / B 1] * 100$.

$B 1$ : The solution found by ACOJSP in the case of 3 transporter vehicles, capacity 1 .

B2: The solution found by ACOJSP in the case of 3 transporter vehicles, capacity 2 .

(b)

\begin{tabular}{lccccc}
\hline Instances & $n$ & $m$ & $\begin{array}{c}\text { ACOJSP } \\
(R=3, \text { Cap }=1)\end{array}$ & $\begin{array}{c}\text { ACOJSP } \\
(R=3, \text { Cap }=3)\end{array}$ & Gap (\%) \\
\hline LT13335 & 3 & 5 & 117 & 126 & 7.7 \\
LT14445 & 4 & 5 & 129 & 129 & 0.0 \\
LT15555 & 5 & 5 & 131 & 157 & 19.8 \\
LT23335 & 3 & 5 & 145 & 153 & 5.5 \\
LT24445 & 4 & 5 & 145 & 148 & 2.1 \\
LT25555 & 5 & 5 & 146 & 143 & -2.1 \\
LT33335 & 3 & 5 & 200 & 200 & 0.0 \\
LT34445 & 4 & 5 & 205 & 205 & 0.0 \\
LT35555 & 5 & 5 & 204 & 189 & -0.5 \\
LT43335 & 3 & 5 & 158 & 194 & 19.6 \\
LT44445 & 4 & 5 & 172 & 223 & 12.8 \\
LT45555 & 5 & 5 & 198 & 252 & 12.6 \\
LT53335 & 3 & 5 & 230 & 292 & 9.6 \\
LT54445 & 4 & 5 & 242 & 268 & 20.7 \\
LT55555 & 5 & 5 & 285 & & -6.0 \\
\hline
\end{tabular}

Gap $=[(B 2-B 1) / B 1] * 100$.

$B 1$ : The solution found by ACOJSP in the case of 3 transporter vehicles, capacity 1 .

$B 2$ : The solution found by ACOJSP in the case of 3 transporter vehicles, capacity 3 . 

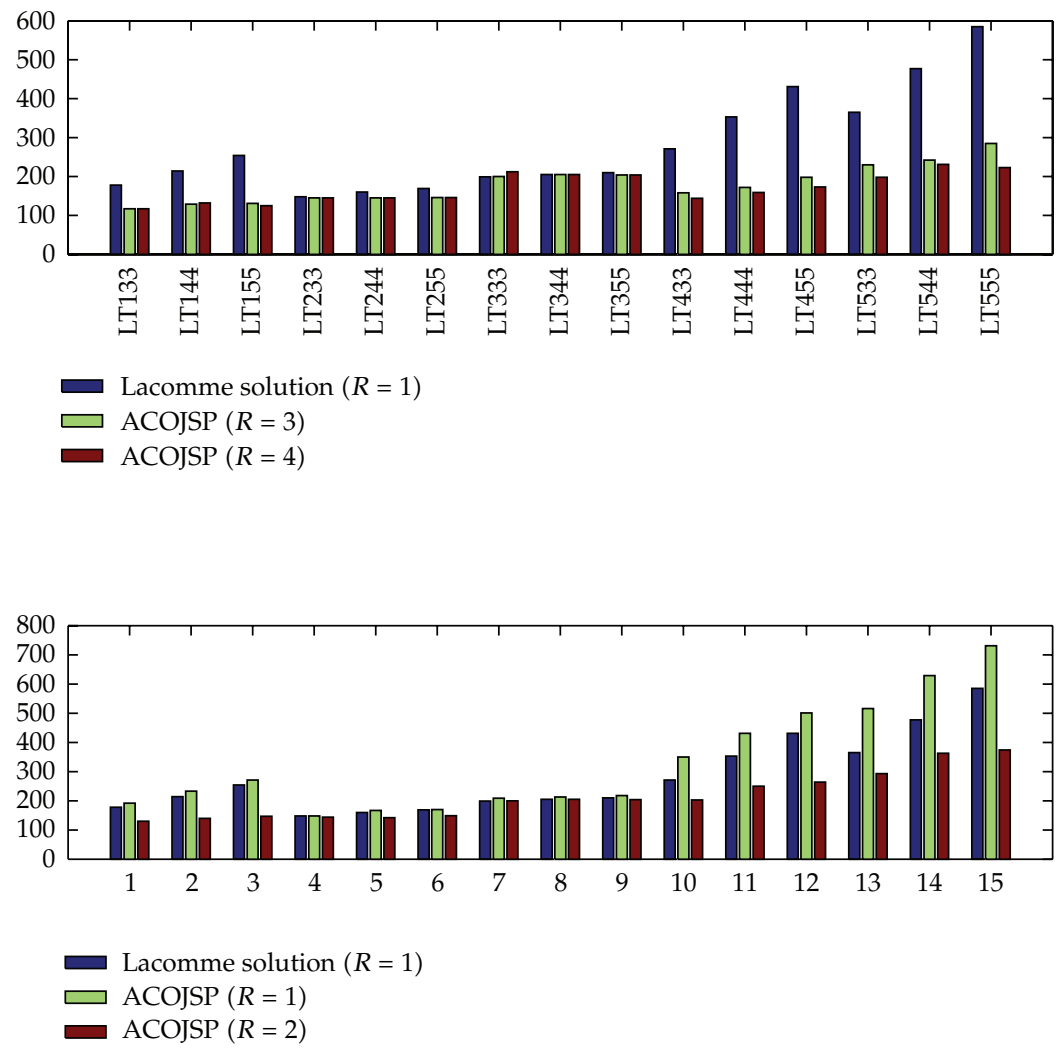

Figure 4: Study of the influence of the number of transporter vehicle in a shop with transport of a set of transporter vehicles.

large instances (LT433-LT555). This difference is justified by the fact that our algorithm is dedicated to several transporter vehicles.

To validate our input management heuristic for the case of single transporter vehicle, we compared it with the proposed Argmin heuristic and four priority rules FIFO, LIFO, SPT, and LPT. The input management heuristic gives the best solutions in most cases.

Table 2 shows the results of this comparison.

\subsubsection{Case of Several Transporter Vehicles}

In this case, we use only the input management heuristic.

By comparing the results obtained by ACOJST in the case of multiple transporter vehicles with the LB given for the case of a single transporter vehicle (see Table 3 and Figure 4), we note that the numerical results show that our algorithm is powerful in the case of several transporter vehicles and especially for instances of great dimension such as LT444, LT555.

\subsection{Transporter Vehicles with a Capacity Equal to 2 and 3}

The Figures 5(a) and 5(b), associated with Tables 4(a) and 4(b), show the behavior of our approach according to the capacity of transfer of the transporter vehicle. Our study is done in 


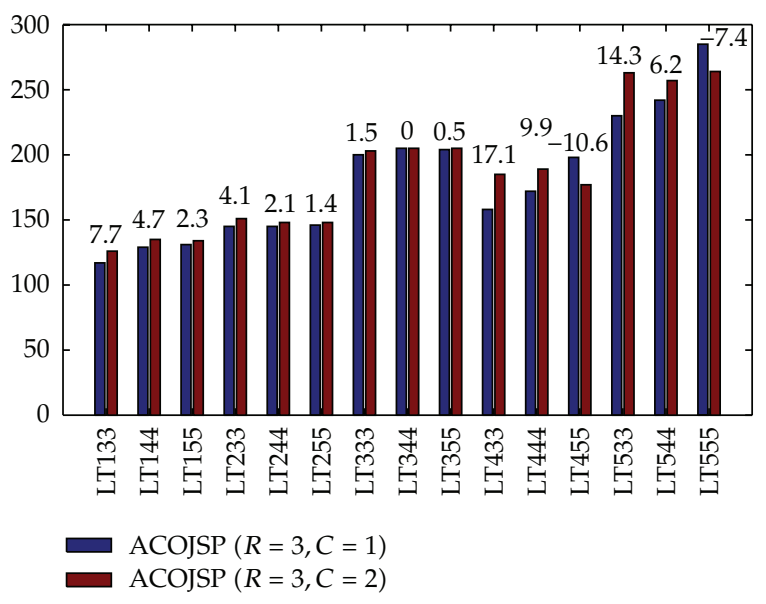

(a)

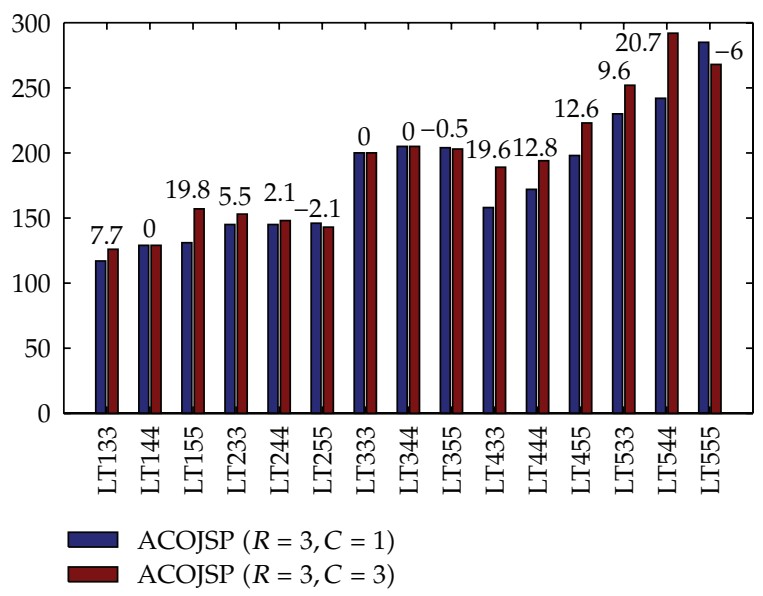

(b)

Figure 5: (a) Study the influence of transfer capacity in the case of a 3 transporter vehicles with 2 capacities. (b) Study the influence of transfer capacity in the case of a 3 transporter vehicles with 3 capacities.

the case of 3 transporter vehicles with 2 and 3 capacities. The results are less good than those obtained using 3 transporter vehicles with capacity 1 and this is due to the constraint of conflict of transporter vehicles, taking into account in the implementation.

\section{Conclusion}

In this work, we studied the general JSSP including temporal constraints related to the transportation of tasks between machines, the capacity of the transporter vehicle, and also their number and the spaces of storage input/output. In the first part of this work, we give a mathematical formulation of the problem and in the second part, we tried to adapt an ant colony algorithm with several heuristics. The work may be extended further by taking into account other real constraints like the management of the topology of stations in the shop and studying the dynamic case. 


\section{References}

[1] A. S. Jain and S. Meeran, “Deterministic job-shop scheduling: past, present and future," European Journal of Operational Research, vol. 113, no. 2, pp. 390-434, 1999.

[2] W. Xia and Z. Wu, "An effective hybrid optimization approach for multi-objective flexible job-shop scheduling problem," Computers E Industrial Engineering, vol. 48, pp. 409-425, 2005.

[3] G. Cavory, R. Dupas, and G. Goncalves, "A genetic approach to solving the problem of cyclic job shop scheduling with linear constraints," European Journal of Operational Research, vol. 161, no. 1, pp. 73-85, 2005.

[4] M. A. Adibi, M. Zandieh, and M. Amiri, "Multi-objective scheduling of dynamic job shop using variable neighborhood search," Expert Systems with Applications, vol. 37, no. 1, pp. 282-287, 2010.

[5] J. Gu, M. Gu, C. Cao, and X. Gu, "A novel competitive co-evolutionary quantum genetic algorithm for stochastic job shop scheduling problem," Computers E Operations Research, vol. 37, no. 5, pp. 927-937, 2010.

[6] J. Chao-Hsien Pan and J.-S. Chen, "Mixed binary integer programming formulations for the reentrant job shop scheduling problem," Computers E Operations Research, vol. 32, no. 5, pp. 1197-1212, 2005.

[7] A. Rossi and G. Dini, "Flexible job-shop scheduling with routing flexibility and separable setup times using ant colony optimization method," Robotics and Computer-Integrated Manufacturing, vol. 23, no. 5, pp. 503-516, 2007.

[8] H. Gröflin and A. Klinkert, “A new neighborhood and tabu search for the blocking job shop," Discrete Applied Mathematic, vol. 157, no. 17, pp. 3643-3655, 2009.

[9] J. Boukachour and A. Benabdelhafid, "Résolution d'un probléme d'ordonnancement de type jobshop par les algorithmes génétiques," in Proceedings of the Troisiéme Conférence Internationale de Mathématiques Appliquées et des Sciences de l'Ingénieur, CIMASI, Casablanca, Maroc, Octobre 2000.

[10] K.-L. Huang and C.-J. Liao, "Ant colony optimization combined with taboo search for the job shop scheduling problem," Computers \& Operations Research, vol. 35, no. 4, pp. 1030-1046, 2008.

[11] A. Soukhal, A. Oulamara, and P. Martineau, "Complexity of flow shop scheduling problems with transportation constraints," European Journal of Operational Research, vol. 161, no. 1, pp. 32-41, 2005.

[12] C.-Y. Lee and Z.-L. Chen, "Machine scheduling with transportation considerations," Journal of Scheduling, vol. 4, no. 1, pp. 3-24, 2001.

[13] J. Hurink and S. Knust, "A tabu search algorithm for scheduling a single robot in a job-shop environment," Discrete Applied Mathematics, vol. 119, no. 1-2, pp. 181-203, 2002.

[14] J. Hurink and S. Knust, "Tabu search algorithms for job shop problems with a single transport robot," European Journal of Operational Research, vol. 162, pp. 99-111, 2005.

[15] P. Lacomme, A. Moukrim, and N. Tchernev, "Simultaneously job input sequencing and vehicle dispatching in a single vehicle AGVS: a heuristic branch and bound approach coupled with a discrete events simulation model," International Journal of Production Research, vol. 43, no. 9, pp. 1911-1942, 2005.

[16] G. El Khayat, A. Langevin, and D. Riopel, “Integrated production and material handling scheduling using mathematical programming and constraint programming," European Journal of Operational Research, vol. 175, no. 3, pp. 1818-1832, 2006.

[17] S. E. Kesen and Ö. F. Baykoç, "Simulation of automated guided vehicle (AGV) systems based on justin-time (JIT) philosophy in a job shop environment," Simulation Modelling Practice and Theory, vol. 15, no. 3, pp. 272-284, 2007.

[18] J. Yuan, A. Soukhal, Y. Chen, and L. Lu, "A note on the complexity of flow shop scheduling with transportation constraints," European Journal of Operational Research, vol. 178, no. 3, pp. 918-925, 2007.

[19] P. Brucker, S. Heitmann, J. Hurink, and T. Nieberg, "Job-shop scheduling with limited capacity buffers," OR Spectrum, vol. 25, no. 2, pp. 151-176, 2006.

[20] A. Caumond, P. Lacomme, A. Moukrim, and N. Tchernev, "A MILP for scheduling problems in an FMS with one vehicle," European Journal of Operational Research, vol. 199, pp. 706-722, 2009.

[21] P. Baptiste, M. Flamini, and F. Sourd, "Lagrangian bounds for just-in-time job-shop scheduling," Computers E Operations Research, vol. 35, no. 3, pp. 906-915, 2008.

[22] R. Cheng, M. Gen, and Y. Tsujimura, "A tutorial survey of job-shop scheduling problems using genetic algorithms-I. Representation," Computers \& Industrial Engineering, vol. 30, no. 40, pp. 983-997, 1996.

[23] R. Abounacer, J. Boukachour, B. Dkhissi, and A. El Hilali Alaoui, "A hybrid Ant Colony Algorithm for the exam timetabling problem," Revue ARIMA, vol. 12, pp. 15-42, 2010. 
[24] G. Bencheikh, J. Boukachour, and A. El Hilali Alaoui, "Improved Ant Colony Algorithm to solve the aircraft landing problem," International Journal of Computer Theory and Engineering, vol. 3, no. 2, pp. 224-233, 2011.

[25] G. Bencheikh, J. Boukachour, A. El Hilali Alaoui, and F. El Khoukhi, "Hybrid method for aircraft landing scheduling based on a Job Shop formulation," International Journal of Computer Science and Network Security, vol. 9, no. 8, 2009. 


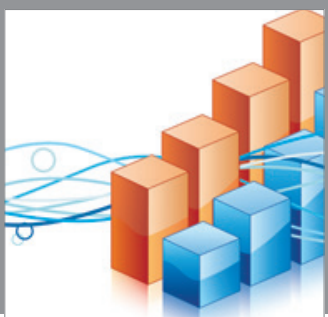

Advances in

Operations Research

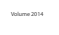

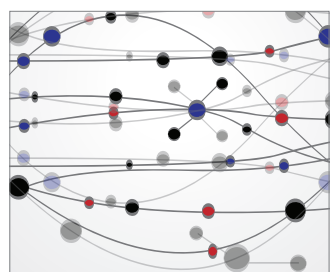

\section{The Scientific} World Journal
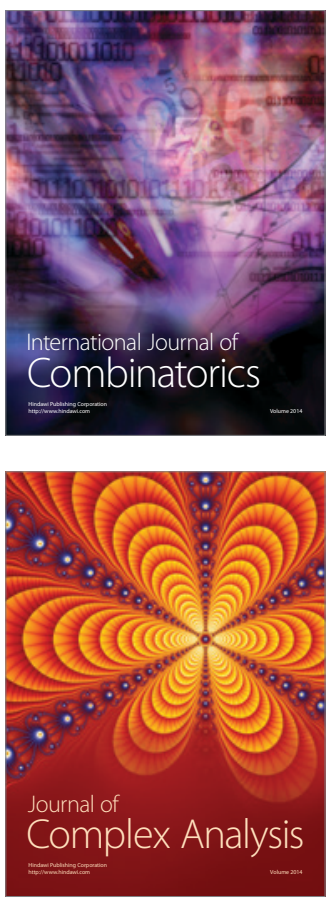

International Journal of

Mathematics and

Mathematical

Sciences
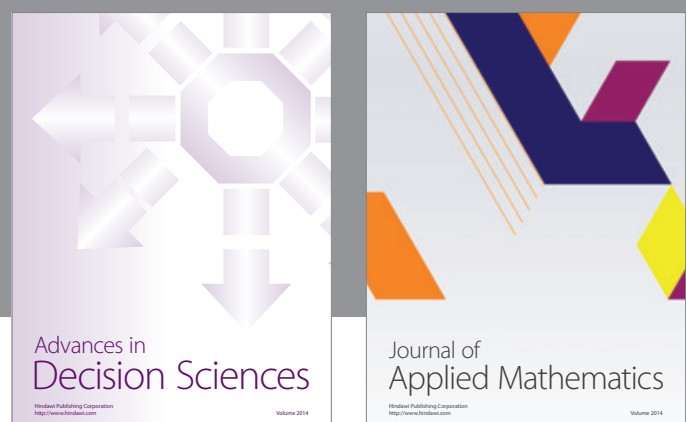

Journal of

Applied Mathematics
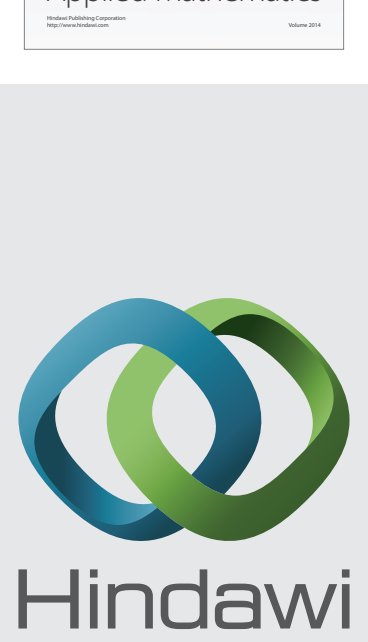

Submit your manuscripts at http://www.hindawi.com
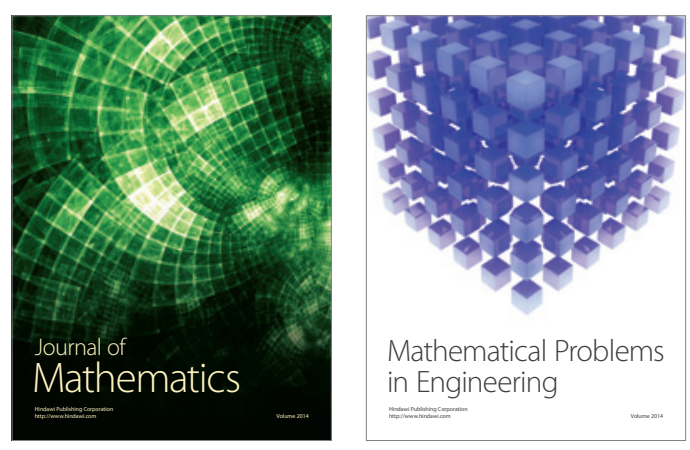

Mathematical Problems in Engineering
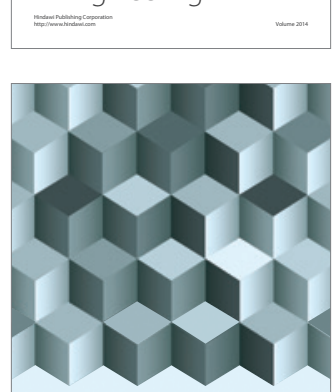

Journal of

Function Spaces
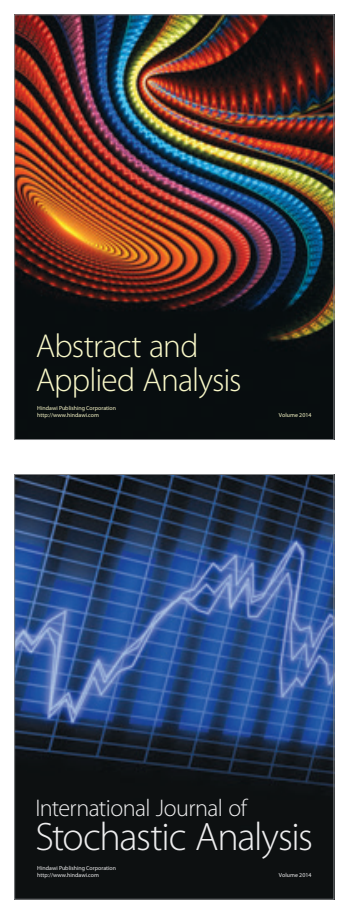

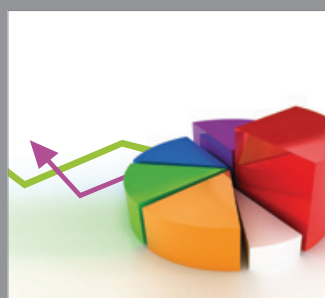

ournal of

Probability and Statistics

Promensencen
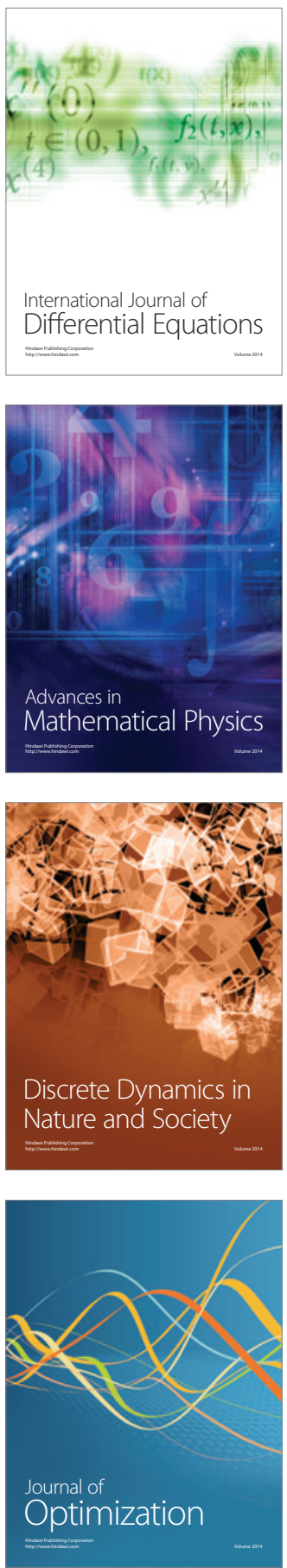